\title{
Performance of bentonite, fly ash and wood ash mixtures as grounding enhancement materials
}

\begin{abstract}
Grounding system is used to divert the lightning surge current, fault current or electromagnetic interference to earth, mainly for safety purposes. An effective grounding system requires low resistance path which could be achieved by treating the surrounding soil with grounding enhancement materials to effectively reduce its earth resistance. Such grounding enhancement materials could be chemical or natural based, where only natural enhancement materials are considered in this study. Six grounding system installations with various ratio of Bentonite, Fly Ash and Wood Ash mixtures were installed at School of Graduate Studies, UPM, and were compared to a Reference grounding system which is without any natural enhancement material added to the vicinity of the ground conductor. The main aim of this work is to evaluate the performance of the natural enhancement material mixtures as grounding enhancement materials. Fall-of-potential method with $62 \%$ rule and $0^{\circ}$ separation angle were employed to measure the grounding system installationsôdaily earth resistances for comparison purposes, for 130 days, i.e. from 12 th Jan 2018 to 22 nd May 2018. The performance of seven grounding system installations considered in this study in the descending order were Bentonite and Wood Ash mixture, 100\% Bentonite, 100\% Wood Ash, Bentonite and Fly Ash mixture, Fly Ash and Wood Ash mixture, 100\% Fly Ash, and Reference grounding systems, with the earth resistance values for the natural enhancement material mixture grounding systems were $20.17 \%, 20.61 \%, 24.17 \%, 25.48 \%, 28.96 \%$, and $34.78 \%$, respectively from the earth resistance value of the Reference grounding system on day-0. This clearly shown that Fly Ash may not be suitable to be used as natural enhancement material for grounding system installation. While, Wood Ash would be a new natural enhancement material as its performance is comparable to Bentonite, the widely used material.
\end{abstract}

Keyword: Grounding system; Natural enhancement material; Bentonite; Fly ash; Wood ash 